Article

\title{
Effect of Number of Variants of Zirconium Hydride on Grain Growth of Zirconium
}

\author{
Bohyun Yoon 1 and Kunok Chang * \\ Department of Nuclear Engineering, Kyung Hee University, Yongin-City 17104, Korea; bohyun.yoon@khu.ac.kr \\ * Correspondence: kunok.chang@khu.ac.kr
}

Received: 31 July 2020; Accepted: 24 August 2020; Published: 27 August 2020

check for

updates

\begin{abstract}
The microstructure characteristics of $\mathrm{Zr}$-hydride in $\mathrm{Zr}$ are important concerns in metallurgy and nuclear engineering. In particular, it is known that the correlation between hydride and the grain boundary microstructure has a great influence on properties. In this study, a phase-field model was used to evaluate evolutions of the fractions of intra-granular and inter-granular hydride and multi-contacted hydride according to the number of structural variants of $\delta$-hydride in the 3D system. The effect of the numbers of crystallographic variants of hydride on grain growth kinetics was also analyzed. We found that the pinning effect in 3D is minimized when hydrides have one crystallographic variant, which is contradictory observation with the 2D case. With grain structures with comparable average grain radii and quantities, we found that the fraction of the intra-granular and inter-granular hydrides increase as the number of crystallographic variants increases.
\end{abstract}

Keywords: zirconium hydride; grain growth; phase-field method

\section{Introduction}

Zirconium $(\mathrm{Zr})$ is a key material in nuclear engineering because of its small neutron absorption cross-section and excellent oxidation resistance [1-3]. The main risk of Zr-based materials in the nuclear system is hydride-induced degradation, such as hydride precipitation [4], hydride reorientation [5-7] and delayed hydride cracking [8]. Therefore, it is extremely important to understand the interaction between $\mathrm{Zr}$ species and their hydrides to ensure the safety of nuclear systems. In this study, we investigated the interaction between zirconium hydride and the grain boundary of zirconium, which can play a significant role in determining the microstructure of the hydride containing $\mathrm{Zr}$. The grain boundary-precipitate interaction is generally understood by Zener pinning [9]. The original theory describes the interaction between a spherical particle and an isotropic grain boundary [9]. For the case of $\delta$ zirconium hydride, which is platelet-shaped $[5,10]$, we need to understand the interaction between a grain boundary with highly anisotropically shaped particles. The interaction between a grain boundary and ellipsoidal particles was estimated by an analytic method [11] and numerical simulations [12,13]. For example, the investigation has been done in 2D to evaluate role of the highly anisotropically-shaped second-phase particle in grain growth [12], and it is necessary to verify whether the trend found in 2D also applies to 3D. Additionally, the relationship between hydride and grain boundary (intra-granular and inter-granular hydride) is a important research topic [14], we need to evaluate how the relative relationship between hydride and grain boundary changes over time in 3D. Recently, the role of the platelet-shaped $\mathrm{Zr}$ hydride in the grain growth of $\mathrm{Zr}$ was experimentally investigated [14]. It was found that the ratio of intra- to inter-granular hydrides increases as grain growth proceeds [14]. Since the effects of inter-granular hydrides and intra-granular hydrides differ in determining the properties of zirconium, such as Zr-matrix strengthening [15], investigations are carried out on the microstructural characteristics of polycrystalline $\mathrm{Zr}$ containing $\mathrm{Zr}$ hydrides. In this 
study, we performed 3D phase-field grain growth simulations with platelet-shaped hydrides, and we systematically examined the role of the number of variants of hydrides in determining grain structure.

\section{Simulation Details}

\subsection{Phase-Field Model for Grain Growth with Hydrides}

The multi-order-parameter grain-growth model was adopted in our simulations [16]. The set of non-conserved order parameters indicates the crystallographic orientations of single-phase polycrystalline materials.

$$
\begin{gathered}
\eta_{1}(\mathbf{r}, t), \eta_{2}(\mathbf{r}, t), \ldots, \eta_{P}(\mathbf{r}, t), \\
\frac{\partial \eta_{i}(\mathbf{r}, t)}{\partial t}=-L_{i} \frac{\delta F}{\delta \eta_{i}(\mathbf{r}, t)}, \quad i=1,2, \ldots, P,
\end{gathered}
$$

where $\eta_{i}(i=1,2, \ldots, P)$ is a non-conserved order parameter represented by position (r) and time $(t)$. We evolved only the active parameter (non-zero) [17] using the Ginzburg-Landau equation in Equation (2) for enhanced computational efficiency.

The total free energy function $F$ with inert precipitates is given by [18]

$$
F=\int_{V}\left[\sum_{i}^{P}\left(\frac{\eta_{i}^{4}}{4}-\frac{\eta_{i}^{2}}{2}\right)+\gamma \sum_{i}^{P} \sum_{i \neq j}^{P} \eta_{i}^{2} \eta_{j}^{2}+\epsilon \Phi(\mathbf{r}) \sum_{i=1}^{P} \eta_{i}^{2}+\frac{\kappa_{i}}{2} \sum_{i}^{P}\left(\nabla \eta_{i}\right)^{2}\right] d V .
$$

With Equations (2) and (3), we obtained

$$
\frac{\partial \eta_{i}(\mathbf{r}, t)}{\partial t}=-L_{i}\left(-\eta_{i}+\eta_{i}^{3}+2 \gamma \eta_{i} \sum_{j=1}^{P} \eta_{j}^{2}+2 \epsilon \Phi(\mathbf{r}) \eta_{i}-\kappa_{i} \nabla^{2} \eta_{i}\right), \quad i=1,2, \ldots, Q
$$

$\Phi(\mathbf{r})$ is 1 at the hydride and becomes 0 at the matrix [18]. $\gamma$ determines the interaction between $\eta_{i}$ and other set of order parameter [19] and $L_{i}$ is related with grain boundary mobility $[16,20] . \kappa_{i}$ is the gradient coefficient which determines the grain boundary energy and width $[16,20]$. In our simulations, the specific order parameter was assigned to only one grain [17].

\subsection{Numerical Details}

To discretize the time derivative in Equation (4), the forward-Euler discretization scheme is applied:

$$
\eta_{i}(t+\Delta t)=\eta_{i}(t)+\frac{d \eta_{i}}{d t} \Delta t+O(\Delta t) .
$$

The phenomenological parameters in Equations (2)-(4) were set to $L_{i}=\gamma=1.0$ and $\kappa_{i}=1.0$ in our simulations. We set $\Delta t=0.1$ in Equation (5) and $\Delta x=1.0$ which we need it to calculate the Laplacian in Equation (4). All phenomenological values are non-dimensionalized values and we can convert it to the quantity with unit, once we have detailed information of grain boundary energy, width and grain growth rate of $\mathrm{Zr}$ system. The phenomenological parameters were selected according to the selection of previous studies that quantitatively reproduced ideal grain growth [16,20]. A periodic boundary condition was applied to all directions. $\epsilon$ was set to 1.0 in our simulations. Initially, spherical particles were distributed to randomly chosen locations, and overlap between initial seed grains was not allowed. OpenMP parallelization was applied to efficiently utilize multi-core CPU capability [21]. The simulation cell size was $512 \Delta x \times 512 \Delta y \times 512 \Delta z$, and initially, spherical grains were distributed. Initially, 20,000 grains were distributed, and we performed simulations for 14,000 time steps.

\subsection{Structural Variations and Morphology of $\delta$-Hydride}

There are three structural variants of $\delta$-hydride when it is precipitated on an $\alpha-\operatorname{Zr}$ matrix [22]. We assume that the angle between the platelet hydride plane and the $x y$ plane is $30^{\circ}, 150^{\circ}$ and 
$270^{\circ}$. A hydride is assumed to be ellipsoid, and the ratio of the major axis to the minor axis is 16 . The volume of the hydride was 171 grid points, and the positions were randomly selected. In this study, we investigated how the number of structural variants affects the microstructural characteristics of the matrix. We examined the cases of one, two and three crystallographic variants of hydrides when the volume fractions of hydrides were $3 \mathrm{vol} \%$ and $7 \mathrm{vol} \%$.

\section{Classification of $\delta$-Hydrides in $\alpha-\mathrm{Zr}$ Matrix}

According to the correlation between hydride and the grain boundary, hydride can be divided into two categories: (1) inter-granular and (2) intra-granular. A schematic illustration of two kinds of hydrides is shown in Figure 1.

Figure 1. Illustrations of inter-granular (green) and intra-granular (blue) hydrides in a polycrystalline $\alpha-\mathrm{Zr}$ matrix. Since we assumed a platelet shape (two axes are symmetric), two forms of inter-granular hydrides, left and right in the figure, can be observed on the 2D cross-sections.

To characterize intra-granular hydride, we evaluated the kind of neighboring grains at every voxel of the hydride. The neighboring grain is counted only for grains that account for more than $2 \%$ of the total interface between the precipitate and the matrix. When the number of neighboring grains is 1 , we assume that the precipitate is intra-granular hydride. When the number of neighboring grains is 2 or more than 2, we call it inter-granular hydride and multi-contacted hydride, respectively.

\section{Results And Discussion}

We visualized three grain structures at $14,000 \Delta t$ in the presence of $3 \mathrm{vol} \%$ hydrides with 1,2 and 3 crystallographic variants in Figures 2-4.

We plotted the fraction of intra-granular (one neighboring grain), inter-granular (two neighboring grains) and multi-contacted (more than two neighboring grains) hydride in the simulations. The driving force of the Zener pinning is the reduction of the interfacial area of the grain boundary in polycrystalline materials [23]; therefore, the inter-granular precipitate is generally more favorable compared with the intra-granular precipitate. However, the global minimum state in terms of the free energy is a one-grain state (no grain boundary), and it is not straightforward to define which state is more favorable between the pinned state, the grain boundary contacting the precipitate, and the unpinned state. 


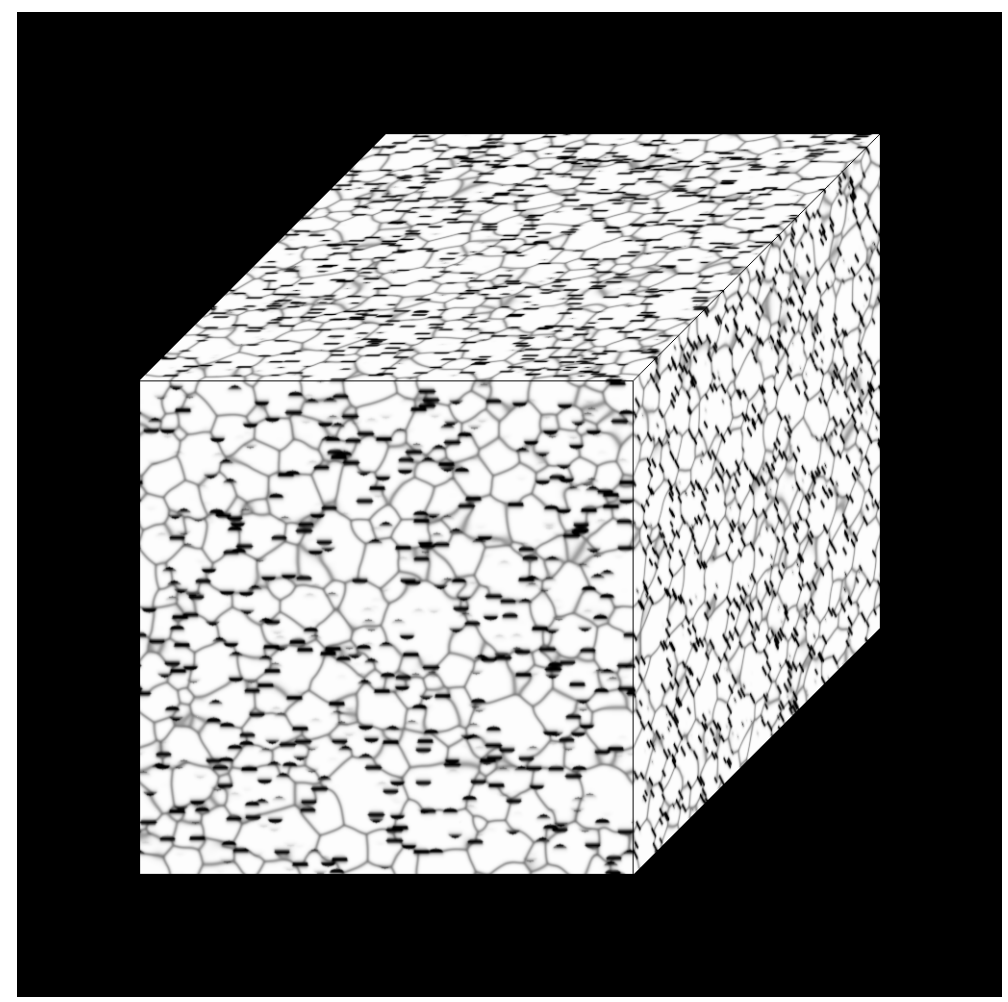

Figure 2. Grain structure with 3 vol\% volume fraction at 14,000 $\Delta t$. All hydrides are aligned along one direction (one crystallographic variant). The system contains 3357 grains, and the average grain radius is 15.799. The volume of each hydride is the same.

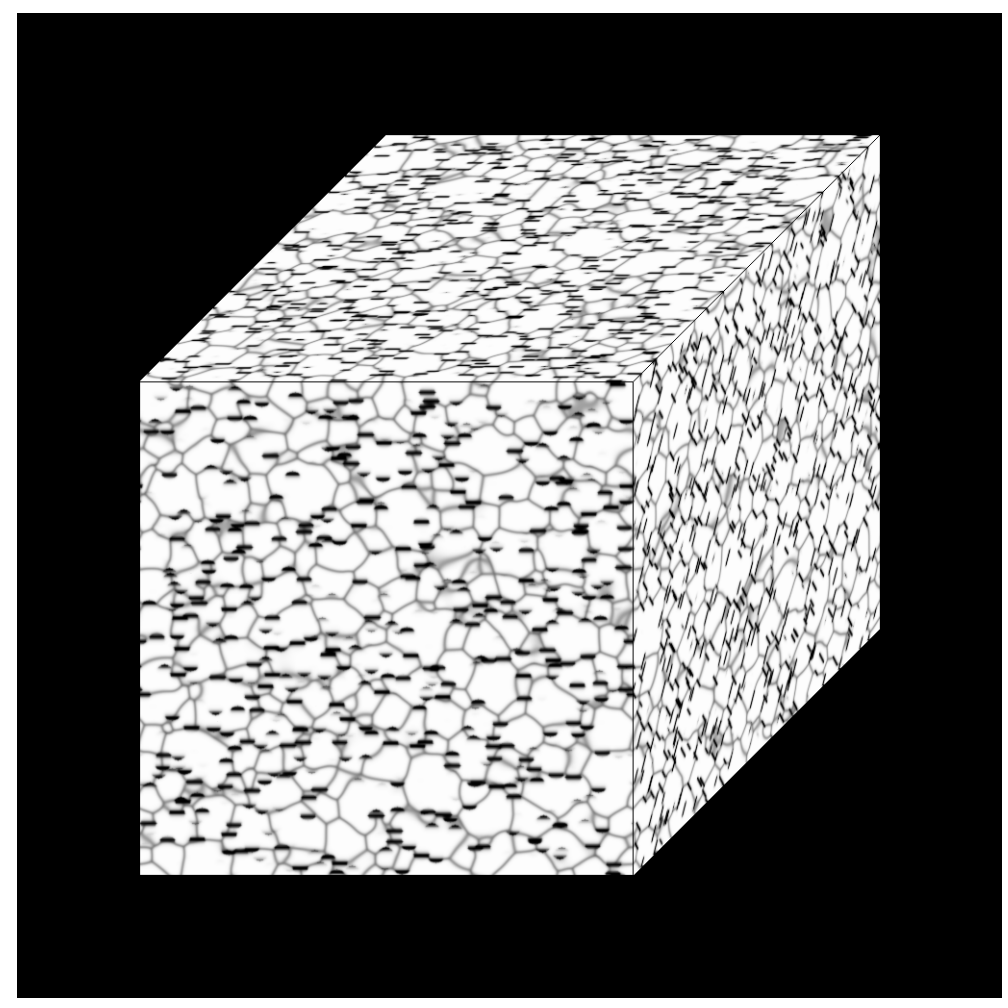

Figure 3. Grain structure with 3 vol\% volume fraction at 14,000 $\Delta t$. The hydrides are aligned along two directions (two crystallographic variants) and the portions of each variant are equal. The system contains 3897 grains and average grain radius is 14.953 . The volume of every hydride is same. 


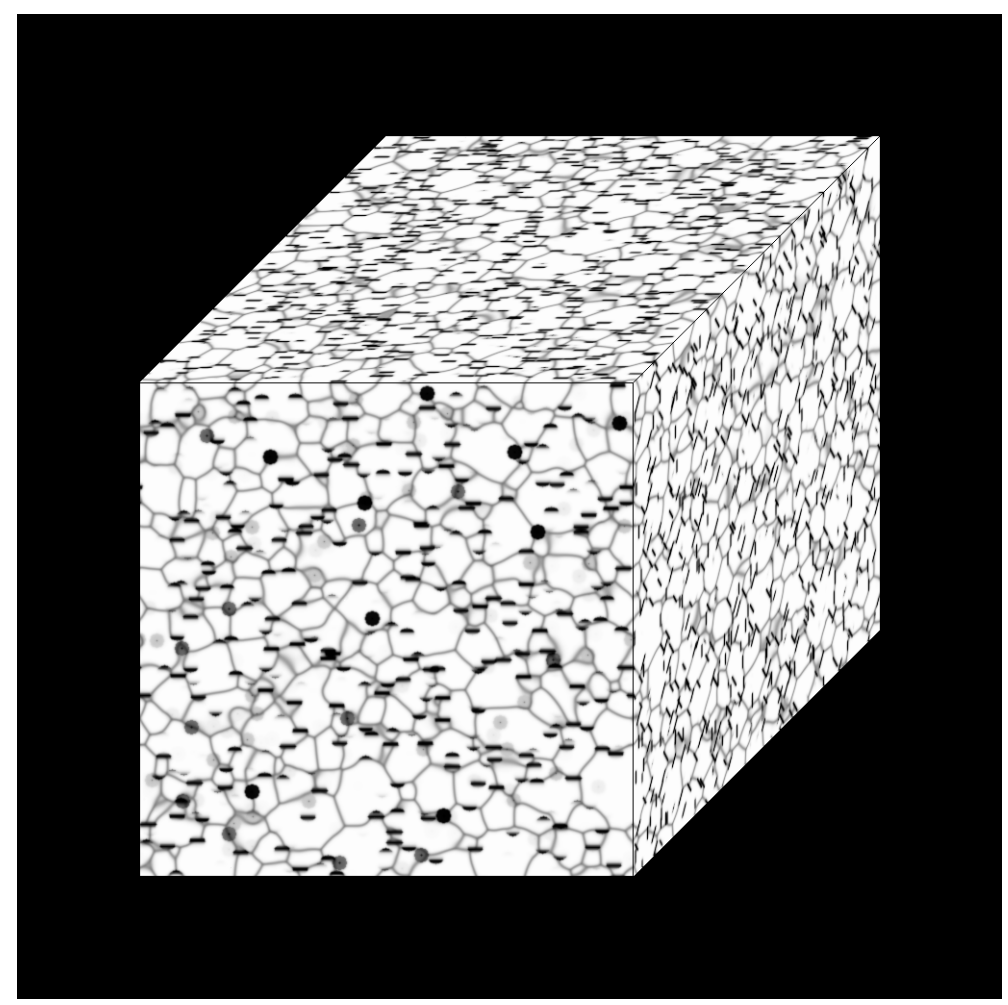

Figure 4. Grain structure with 3 vol $\%$ volume fraction at $14,000 \Delta t$. The hydrides are aligned along three directions (three crystallographic variants), and the portions of each variant are equal. The system contains 3669 grains, and the average grain radius is 15.246 . The volume of every hydride is the same.

In our simulations, the majority of hydrides were evaluated as multi-contacted hydrides; their fractions decrease as grain growth proceeds, and the fractions of the inter-granular and intra-granular hydrides increase as grain size increases in Figures 5 and 6 . For the 3 vol $\%$ and 7 vol\% cases, the fractions of the inter-granular and the intra-granular hydrides have largest values when 3 crystallographic variants cases. On the other hand the minimum fractions of inter- and intra-granular hydrides are shown in 1 variant cases at early stage; however, 2 variants cases have the minimum values at later stage. When all hydride is aligned along 1 direction (1 variant), it is the least efficient in terms of suppressing grain growth among 1-, 2- and 3-variant cases in Figure 7 for $3 \mathrm{vol} \%$ and $7 \mathrm{vol} \%$ cases. Consistently, the grains are eliminated most rapidly when number of structural variants is 1 for $3 \mathrm{vol} \%$ and $7 \mathrm{vol} \%$ cases in Figure 8. In 2D systems, the pinning effect decreased as the direction of the needle-shaped particle increased [12]. In 3D case, the correlation between number of variants and how effectively retard grain growth is not analyzed as clearly as in 2D case. One of the reasons of that is, in 3D, since the crystallographic variant is defined using two angles, the correlation between the more general directionality (crystallographic variants) and the pinning effect should be assessed in a more general situation than the $\mathrm{Zr}-\mathrm{H}$ system. Regardless, the tentative conclusion that can be obtained through the simulations considering three structural variants of Zirconium hydride in 3D is, the particle pinning effect due to precipitation aligned in 1 direction is notably less than in 2-and 3-directions aligned cases. Even when platelet-shaped precipitates are aligned in a random direction, we expect that the particle pinning effect will not be significantly different in the 2- and 3- variants cases. Experimentally, the inter-granular hydride ratio increased as grain growth progressed [14], and this is considered to be the means of classifying a 3D multi-contacted hydride as inter-granular or intra-granular in the 2D cross-section. 
3 vol\% of hydrides

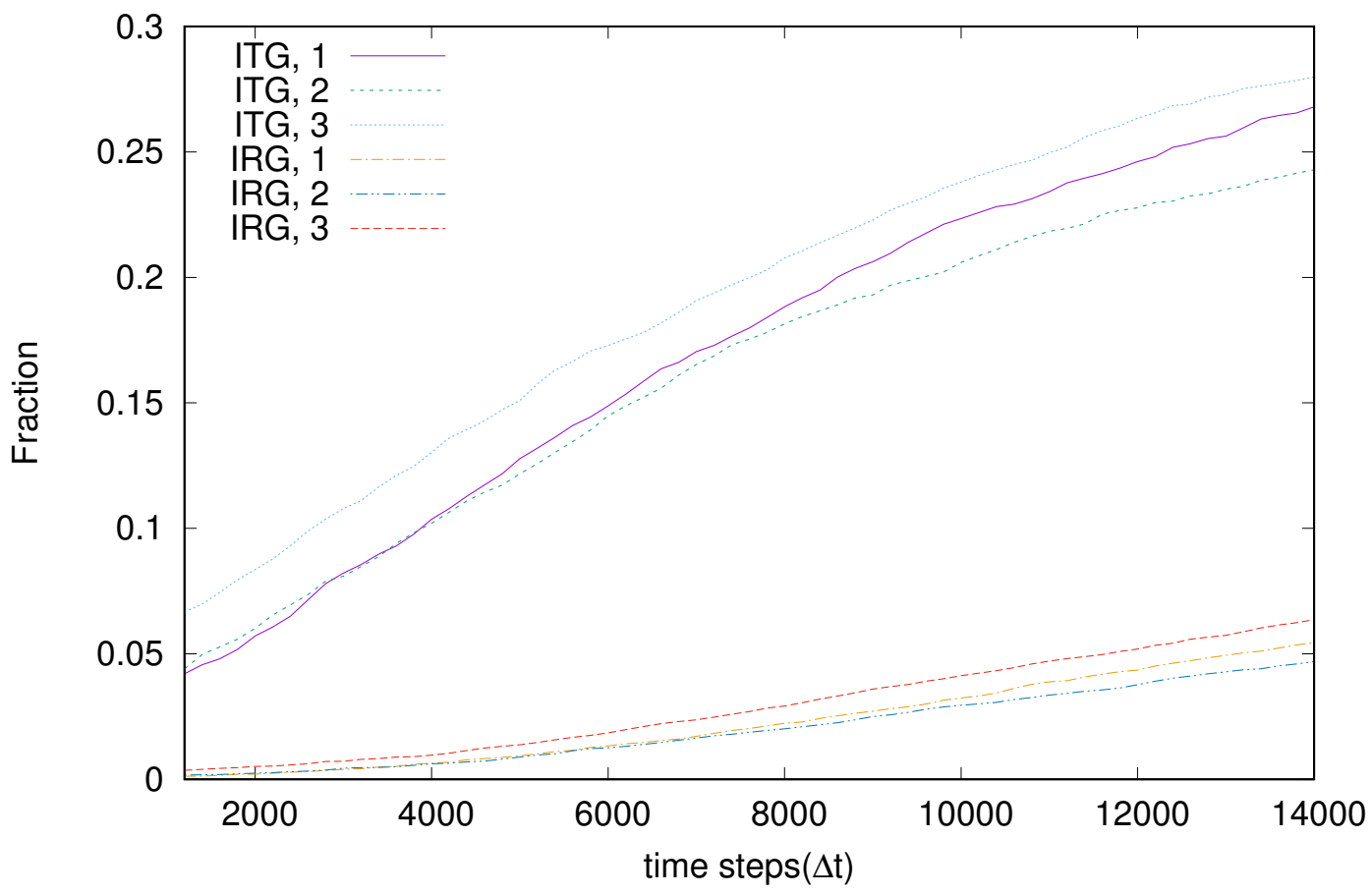

Figure 5. Plot of fractions of intra-granular and inter-granular hydrides with respect to time in the presence of $3 \mathrm{vol} \%$ hydrides. The fractions of hydrides with 1, 2 and 3 structural variants cases are plotted. ITG and IRG stand for Inter-granular, Intra-granular, respectively and the numbers next to comma indicate the number of structural variants.

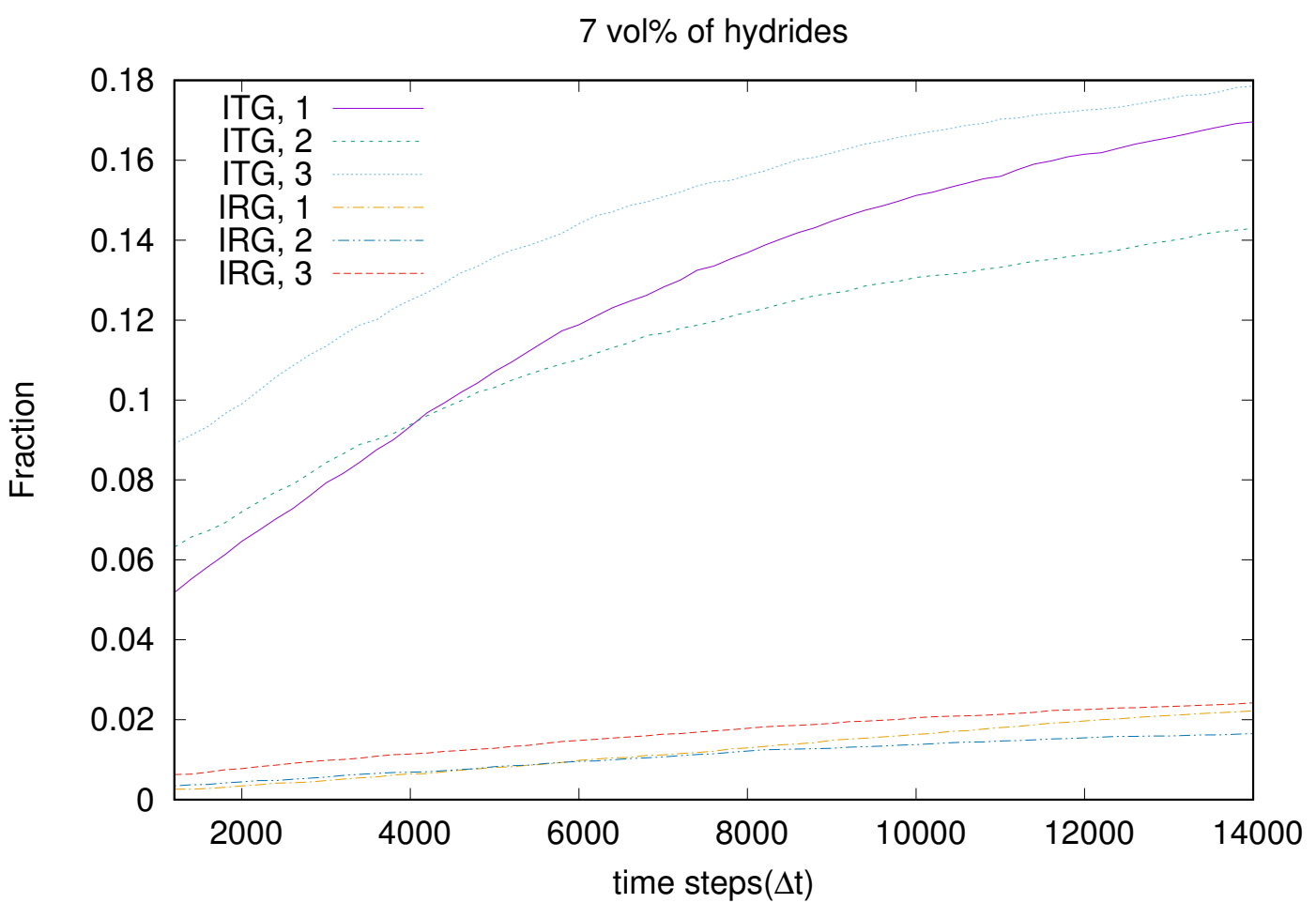

Figure 6. Plot of fractions of intra-granular and inter-granular hydrides with respect to time in the presence of $7 \mathrm{vol} \%$ hydrides. The fractions of hydrides with 1, 2 and 3 structural variants cases are plotted. ITG and IRG stand for Inter-granular, Intra-granular, respectively and the numbers next to comma indicate the number of structural variants. 
We also analyzed the effect of crystallographic variants with comparable average grain radii and grain numbers; for example, in a comparison between one-variant 3 vol\% hydride with an average grain radius of 14.910 and with 3908 grains at $11,600 \Delta t$ and three-variant 3 vol\% hydride with an average grain radius of 14.963 and with 3876 grains at 13,000 $\Delta t$, the fractions of inter-granular and intra-granular hydrides are larger in the three-variant case rather than the one-variant case. For the case of the two-variant $3 \mathrm{vol} \%$ hydride with an average grain radius of 14.953 and with 3897 grains at $14,000 \Delta t$, the fractions of inter-granular and intra-granular hydrides have values between those of one-variant $3 \mathrm{vol} \%$ hydride at 11,600 $\Delta t$ and three-variant $3 \mathrm{vol} \%$ hydride at $13,000 \Delta t$. For the 7 vol $\%$ case, we found consistent trends and concluded that fractions of intra-granular and inter-granular hydrides increase as the number of hydride variants increases for comparable average grain radii and quantities. In this study, only the movement of the grain boundary was considered when hydride was already precipitated, but it was predicted that hydride would be better precipitated in the grain boundary with high energy $[15,24]$. Especially, according to a previous study that analyzed the precipitation of intergranular and intragranular hydride using classical nucleation theory [15], the inhomogeneity of grain boundary energy was judged as an important variable in determining the hydride precipitation behavior. However, the evolution in the microstructure of the $\mathrm{Zr}$ alloy are found in heat treatment of $550{ }^{\circ} \mathrm{C}$ or higher [4] and typical heat treatment temperature of $\mathrm{Zr}$ alloys for grain growth is $700{ }^{\circ} \mathrm{C}[25,26]$. Therefore, precipitation of hydride at this range of temperature $\left(\sim 700{ }^{\circ} \mathrm{C}\right)$ was neglected in this study. Further studies are needed in the future to determine how hydride precipitation at grain boundary affects microstructure changes such as grain growth or recrystallization. Also, it is worthy to investigate effect of grain boundary energy inhomogeneity in hydride precipitation behavior.

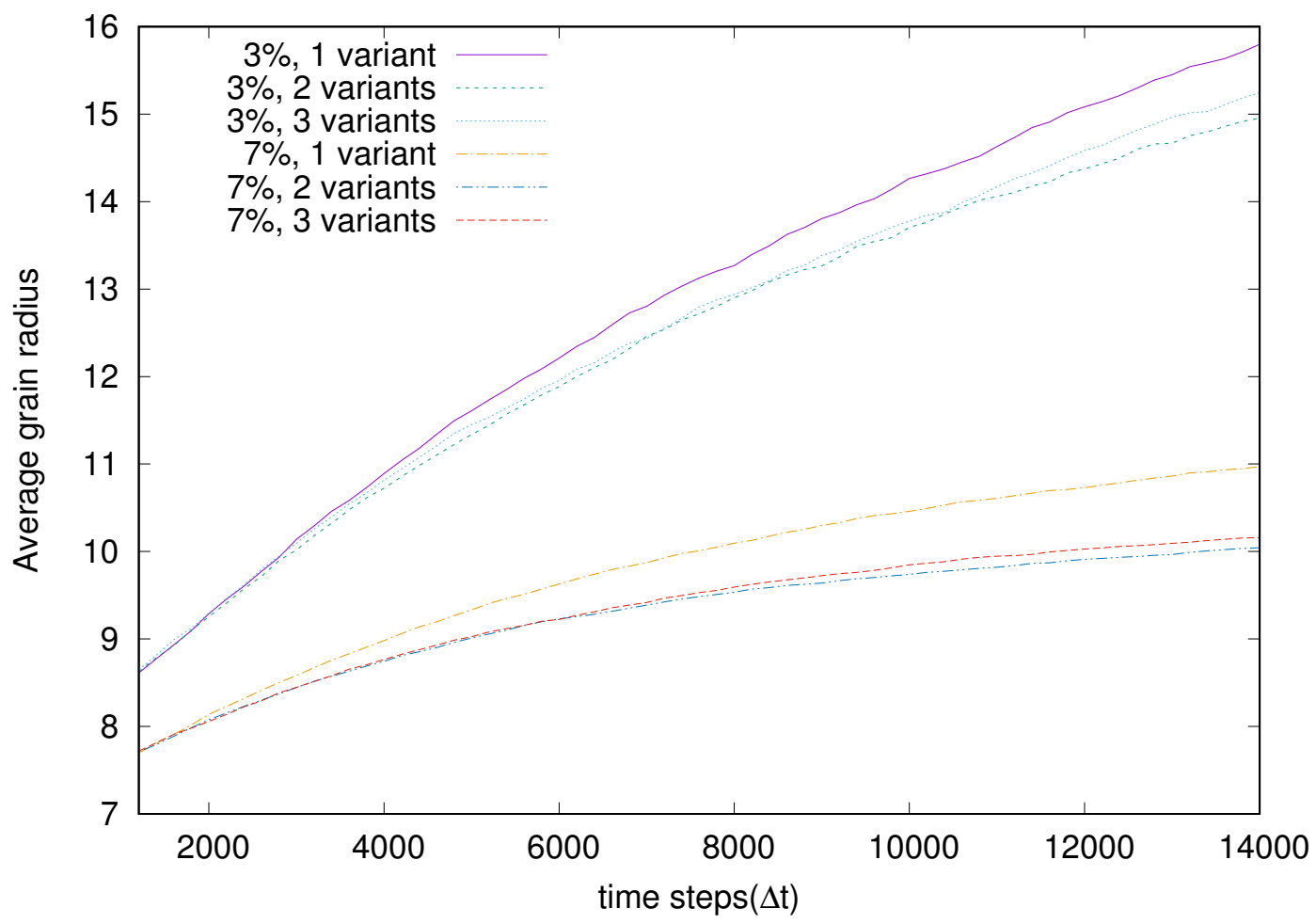

Figure 7. Average grain radius with respect to time when $3 \mathrm{vol} \%$ hydrides and $7 \mathrm{vol} \%$ hydrides are involved. We plotted the values for the cases of 1,2 and 3 structural variants of the hydrides. 


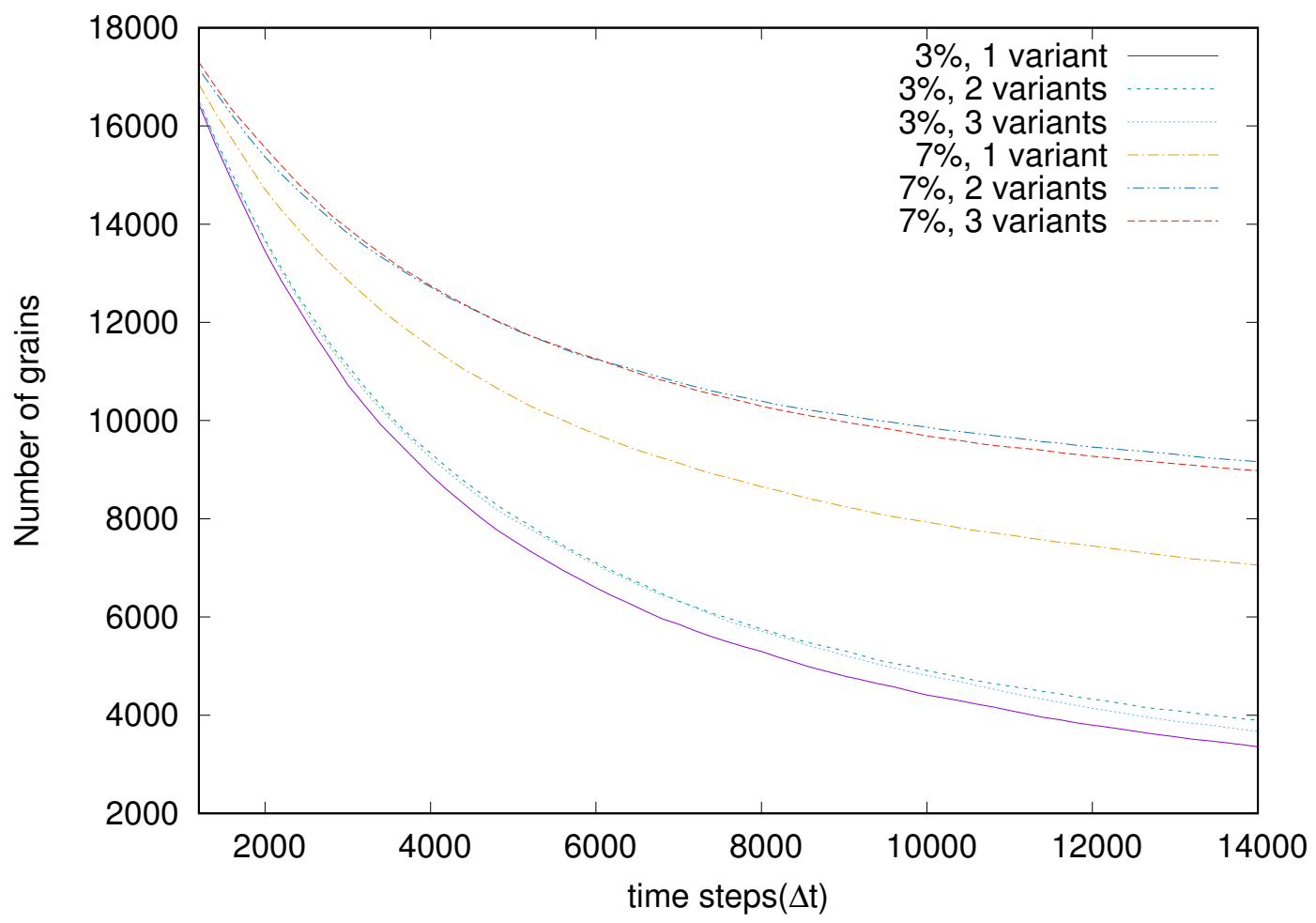

Figure 8. Number of grains with respect to time when 3 vol\% hydrides 7 vol\% hydrides are involved. We plotted the values for the cases of 1, 2 and 3 structural variants of the hydrides.

\section{Conclusions}

In 3D systems including platelet-shaped hydride, a set of grain growth simulations were performed using the phase-field model to evaluate the effect of number of crystallographic variants on microstructure changes. The fractions of inter-granular and intra-granular hydrides increase as simulations proceeds. The relative quantities of the inter-granular and intra-granular hydrides are maximized when 3 crystallographic variants are considered among cases of 1,2 and 3 variants. Unlike the 2D case, a tendency to not retard grain growth efficiently when the number of variants is 1 was also found in 3D.

Author Contributions: Conceptualization, K.C.; methodology, K.C.; software, B.Y.; validation, K.C., B.Y.; formal analysis, B.Y.; investigation, B.Y.; resources, B.Y.; data curation, B.Y.; writing-original draft preparation, K.C.; writing-review and editing, K.C.; visualization, B.Y.; supervision, K.C.; project administration, K.C.; funding acquisition, K.C. All authors have read and agreed to the published version of the manuscript.

Funding: This work has supported by the National Research Foundation of Korea (NRF) grant funded by the Korea government (MSIT) (NRF-2018R1C1B5040669). This work was also supported by the "Human Resources Program in Energy Technology" of the Korea Institute of Energy Technology Evaluation and Planning (KETEP), granted financial resources from the Ministry of Trade, Industry \& Energy, Republic of Korea (No. 20184030202170).

Conflicts of Interest: The authors declare no conflict of interest.

\section{References}

1. Golden, L.B.; Lane, I.R.; Acherman, W.L. Corrosion resistance of titanium, zirconium, and stainless steel. Ind. Eng. Chem. 1952, 44, 1930-1939. [CrossRef]

2. Lustman, B.; Kerze, F. The Metallurgy of Zirconium; McGraw-Hill: New York, NY, USA, 1955; Volume 4.

3. Filburn, T.; Bullard, S. Nuclear Fuel, Cladding, and the "Discovery" of Zirconium. In Three Mile Island, Chernobyl and Fukushima; Springer: Berlin, Germany, 2016; pp. 105-114.

4. Kim, S.S.; Lim, S.; Ahn, D.H.; Lee, G.G.; Chang, K. Effect of Inhomogeneous Nucleation of Hydride at $\alpha / \beta$ Phase Boundary on Microstructure Evolution of $\mathrm{Zr}-2.5 \mathrm{wt} \% \mathrm{Nb}$ Pressure Tube. Met. Mater. Int. 2019, 25, 838-845. [CrossRef] 
5. Shin, W.; Chang, K. Phase-field modeling of hydride reorientation in zirconium cladding materials under applied stress. Comput. Mater. Sci. 2020, 182, 109775. [CrossRef]

6. Colas, K.B.; Motta, A.T.; Daymond, M.R.; Almer, J.D. Effect of thermo-mechanical cycling on zirconium hydride reorientation studied in situ with synchrotron X-ray diffraction. J. Nucl. Mater. 2013, 440, 586-595. [CrossRef]

7. Kim, J.S.; Kim, Y.J.; Kook, D.H.; Kim, Y.S. A study on hydride reorientation of Zircaloy-4 cladding tube under stress. J. Nucl. Mater. 2015, 456, 246-252. [CrossRef]

8. Puls, M.P. Review of the thermodynamic basis for models of delayed hydride cracking rate in zirconium alloys. J. Nucl. Mater. 2009, 393, 350-367. [CrossRef]

9. Smith, C.S. Grains, phases, and interfaces: An introduction of microstructure. Trans. Metall. Soc. AIME 1948, 175, 15-51.

10. Westlake, D. The habit planes of zirconium hydride in zirconium and zircaloy. J. Nucl. Mater. 1968, 26, 208-216. [CrossRef]

11. Nes, E.; Ryum, N.; Hunderi, O. On the Zener drag. Acta Metall. 1985, 33, 11-22. [CrossRef]

12. Chang, K.; Feng, W.; Chen, L.Q. Effect of second-phase particle morphology on grain growth kinetics. Acta Mater. 2009, 57, 5229-5236. [CrossRef]

13. Chang, K.; Chen, L.Q. Quantitative evaluation of particle pinning force on a grain boundary using the phase-field method. Model. Simul. Mater. Sci. Eng. 2012, 20, 055004. [CrossRef]

14. El Chamaa, S.; Patel, M.; Davies, C.; Wenman, M.R. The Effect of Grain Boundaries and Second-Phase Particles on Hydride Precipitation in Zirconium Alloys. MRS Adv. 2018, 3, 1749-1754. [CrossRef]

15. Qin, W.; Kumar, N.K.; Szpunar, J.; Kozinski, J. Intergranular $\delta$-hydride nucleation and orientation in zirconium alloys. Acta Mater. 2011, 59, 7010-7021. [CrossRef]

16. Chen, L.Q.; Yang, W. Computer simulation of the domain dynamics of a quenched system with a large number of nonconserved order parameters: The grain-growth kinetics. Phys. Rev. B 1994, 50, 15752. [CrossRef] [PubMed]

17. Vedantam, S.; Patnaik, B. Efficient numerical algorithm for multiphase field simulations. Phys. Rev. E 2006, 73, 016703. [CrossRef]

18. Moelans, N.; Blanpain, B.; Wollants, P. A phase field model for the simulation of grain growth in materials containing finely dispersed incoherent second-phase particles. Acta Mater. 2005, 53, 1771-1781. [CrossRef]

19. Chang, K.; Moelans, N. Effect of grain boundary energy anisotropy on highly textured grain structures studied by phase-field simulations. Acta Mater. 2014, 64, 443-454. [CrossRef]

20. Krill Iii, C.; Chen, L.Q. Computer simulation of 3-D grain growth using a phase-field model. Acta Mater. 2002, 50, 3059-3075. [CrossRef]

21. Dagum, L.; Menon, R. OpenMP: An industry standard API for shared-memory programming. IEEE Comput. Sci. Eng. 1998, 5, 46-55. [CrossRef]

22. Heo, T.W.; Colas, K.B.; Motta, A.T.; Chen, L.Q. A phase-field model for hydride formation in polycrystalline metals: Application to $\delta$-hydride in zirconium alloys. Acta Mater. 2019, 181, 262-277. [CrossRef]

23. Doherty, R. Role of interfaces in kinetics of internal shape changes. Met. Sci. 1982, 16, 1-14. [CrossRef]

24. Szpunar, J.A.; Qin, W.; Li, H.; Kumar, N.K. Roles of texture in controlling oxidation, hydrogen ingress and hydride formation in Zr alloys. J. Nucl. Mater. 2012, 427, 343-349. [CrossRef]

25. Chun, Y.; Hwang, S.; Kim, M.; Kwun, S.; Kim, Y. Effect of Mo on recrystallization characteristics of $\mathrm{Zr}-\mathrm{Nb}-(\mathrm{Sn})-\mathrm{Mo}$ experimental alloys. J. Nucl. Mater. 1999, 265, 28-37. [CrossRef]

26. Hiwarkar, V.; Sahoo, S.; Samajdar, I.; Narasimhan, K.; Krishna, K.M.; Dey, G.; Srivastava, D.; Tewari, R.; Banerjee, $\mathrm{S}$. Annealing of cold worked two-phase $\mathrm{Zr}-2.5 \mathrm{Nb}$-Associated microstructural developments. J. Nucl. Mater. 2009, 384, 30-37. [CrossRef]

(C) 2020 by the authors. Licensee MDPI, Basel, Switzerland. This article is an open access article distributed under the terms and conditions of the Creative Commons Attribution (CC BY) license (http:/ / creativecommons.org/licenses/by/4.0/). 